psychopraxis.neuropraxis $2015 \cdot 18: 113$

DOI 10.1007/s00739-015-0261-2

Online publiziert: 21. Mai 2015

○) Springer-Verlag Wien 2015
Elisabeth Fertl

Abteilungsvorständin der Neurologischen Abteilung, Krankenanstalt Rudolfstiftung, Wien, Österreich

\title{
Der gemeinsame Patient von Neurologie und Psychiatrie
}

\section{Liebe Leserinnen und Leser!}

Sie halten nun druckfrisch eine neue Ausgabe des interdisziplinären wissenschaftlichen Journals „psychopraxis.neuropraxis“ in Händen. Nach umfangreichen Vorarbeiten des Herausgeberteams, der Verlagsmitarbeiter und der Autoren ist es wieder gelungen, ein sehr breites Spektrum an interdisziplinären Themen zwischen Neurologie und Psychiatrie aufzugreifen.

Epilepsie ist eine sehr häufige chronische neurologische Erkrankung, die Personen jeden Geschlechts und jeden Alters betreffen kann. Relativ unbekannt ist, dass bei Epilepsie-Patienten sehr häufig eine psychiatrische Komorbidität besteht. Dabei ist das diagnostische Spektrum der psychischen Symptome in der Epilepsie sehr breit. Aktuell greifen wir das Thema Substanzabusus und Epilepsie auf, weil in der Patientenversorgung hier oft eine enge Verflechtung zwischen den beiden Fächern besteht. In einem umfassenden Übersichtsartikel beschreibt Dr. Julia Höfler, SALK Salzburg, die diagnostischen und therapeutischen Herausforderungen bei solchen Patienten. Darüber hinaus wird diese Themenkombination durch einen Fallbericht aus meiner Abteilung praxisnahe illustriert.

Die Behandlung von Patienten mit bipolaren Störungen mit Lithium als Phasenprophylaxe ist seit Jahrzehnten eine Säule der Psychiatrie. Dennoch gerät zunehmend das Wissen um Vor- und Nachteile dieser erprobten Therapiesäule in Vergessenheit. Vor allem ist unbekannt, dass bei älteren Personen, das heißt bei Senioren über 60, eine Dosisadaptation an die veränderten Stoffwechselbedingungen des Alters dringend erforderlich ist. Wenn eine derartige Dosisanpassung von Lithium aus Altersgründen oder wegen einer
Komedikation nicht vorgenommen wird, kann es zu einer akuten oder auch chronischen Lithium-Intoxikation kommen. Diese Problemstellung wird ausführlich und fachkundig im Artikel von Dr. Armand Hausmann, MedUni Innsbruck, beschrieben. Es zeigt sich hier ein ganzes Kontinuum von neurologischen Symptomen der Lithium-Intoxikation, beginnend bei Tremor über epileptische Serienanfälle bis hin zum lebensbedrohlichen Koma, das auch in der Ausbildung des fachärztlichen Nachwuchses gelehrt werden muss. Somit sind bei einer LithiumTherapie psychiatrischer Patienten langfristig beide Fächer - Neurologie ebenso wie die Psychiatrie - gefragt. Gerade in Abteilungen mit neurologischen Notfallsaufnahmen finden sich immer wieder $\mathrm{Pa}$ tienten mit akuter Lithium-Intoxikation, die dann nur durch Dialyse rasch behandelt werden können. Ohne interdisziplinäre Notfallversorgung ist hier die Psychiatrie alleine hilflos. Aber auch chronische subtile Symptome einer Lithium-Intoxikation werden zunehmend spannender.

Last, but not least wird in diesem Heft ausführlich auf den Stellenwert und die verschiedenen Settings der psychosomatischen Diagnostik und Therapie eingegangen. Die Existenz einer eigenständigen Psychosomatik ergab sich im historischen Kontext aus der Organspezialisierung der Medizin. Die Fachärzte der Organfächer der Medizin erhielten in den vergangenen 100 Jahren leider keine praktische psychiatrische Grundausbildung. Dennoch bemerkten Kollegen dieser Fachrichtungen, dass ihre Patienten auch psychische Beschwerden haben und dass in vielen Fällen chronisch organische Beschwerden psychogenen Ursprungs sind. Aus dieser „Fachlücke“ heraus entstand dann die Psychosomatik als Querschnittsmaterie. Ältere Daten zeig- ten ja, dass Patienten mit psychisch verursachten Organbeschwerden im Mittel bis zu 11 Jahre ihren Weg durch das Gesundheitswesen nehmen, bevor sie lösungsorientiert diagnostiziert und behandelt werden. Diesen für alle Beteiligten mühseligen, gefährlichen und kostenintensiven Weg zum richtigen Ziel versuchen die heutigen Ansätze der Psychosomatik zu verkürzen. Der Beschreibung dieser Einrichtungen ist in diesem Heft breiter Raum gewidmet. Somit wünsche ich Ihnen, liebe Leserinnen und Leser viel Freude bei der Lektüre dieses Heftes, interessante Anregungen und Inspirationen für Ihre klinische Tätigkeit.

Mit freundlichen Grüßen

Prim. Univ.-Doz. Dr. Elisabeth Fertl

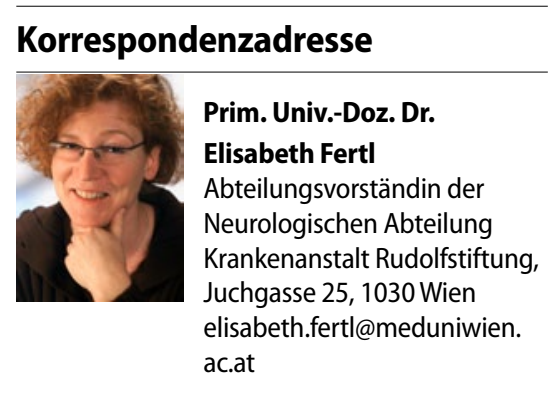

